\title{
ON GROUPS OF FINITE HEIGHT
}

\author{
STEPHEN J. PRIDE
}

(Received 31 May 1978)

Communicated by $\mathrm{H}$. Lausch

\begin{abstract}
In a previous paper, "The concept of "largeness" in group theory', a partial order was defined on the class of infinite groups, and this partial order was seen to give some precision to our intuitive notions of what it means for one infinite group to be 'larger' than another. The aim of this paper is to look more closely at groups which are 'low down' in this partial order, and to examine the interplay between large properties of groups and finiteness conditions in group theory.
\end{abstract}

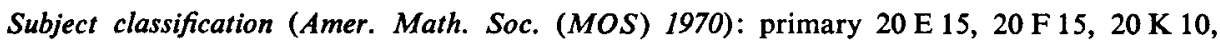
$20 \mathrm{~K} \mathrm{25}$; secondary $20 \mathrm{E} 10$.

\section{Introduction}

This paper is a continuation of the study of the concept of largeness in group theory which was begun in Pride (to appear). Familiarity with that paper will be assumed throughout.

Theideas introduced in Pride (to appear) have already provided a useful framework for investigating groups thought of as being 'large'-see Baumslag and Pride (1978). The main aim of this paper is to look at the opposite extreme and to examine the interplay between large properties of groups and finiteness conditions in group theory. This is obviously of intrinsic interest in relation to the ideas developed in Pride (to appear). More importantly, however, it puts the study of finiteness conditions into a wider context, and therefore leads to a different, and hopefully profitable, way of looking at things.

A brief illustration can be given immediately of the way in which finitary aspects of infinite group theory are thrown into a new light by a study of large properties. In the theory of abelian groups, groups of the following three types play a significant role as 'building blocks': quasicyclic groups, infinite cyclic groups, countable 
elementary groups. Now, as will be seen later, there is an obvious subdivision of the class $\mathscr{M}$ of minimal groups into three disjoint subclasses, $\mathscr{K}_{1}, \mathscr{H}_{2}^{1}, \mathscr{H}_{2}^{2}$. It turns out that an abelian group is minimal if and only if it is a finite extension of a group of one of the three types mentioned above; moreover, quasicyclic groups lie in $\mathscr{M}_{1}$, infinite cyclic groups lie in $\mathscr{H}_{2}^{1}$ and countable elementary groups lie in $\mathscr{H}_{2}^{2}$.

Some of the interplay between large properties of groups and finiteness conditions in group theory can already be seen in Pride (to appear). For example, any group satisfying the finiteness condition 'being just-infinite and having Max-sn' is of finite height. Every finitely generated abelian group has finite height. Any group $G$ which satisfies Max-an (and so, in particular, any group satisfying Max-sn) can have infinite height, although there cannot be a chain $\ldots \prec G_{2} \prec G_{1} \prec G_{0}=G$ (that is $G$ must satisfy Min- $\preccurlyeq$ ). Similarly, any group satisfying Min-an must satisfy Max- $\preccurlyeq$.

In contrast to the results in the previous paragraph, the finiteness conditions 'being residually finite' and 'being finitely generated' are enjoyed by $F_{2}$, which certainly does not satisfy Max- $\preccurlyeq$ or Min- $\preccurlyeq$.

The above examples illustrate exactly what one intuitively expects: really incisive finiteness conditions give groups of finite height, those which are not so incisive give groups satisfying either Max $-\preccurlyeq$ or Min- $\preccurlyeq$ and those which are not very incisive can be satisfied by groups without Max $-\preccurlyeq$ or Min $-\preccurlyeq$.

This paper will concentrate on examining finiteness conditions which lead to groups of finite height.

The early part of the paper (Section 2) is concerned with the class of minimal groups. As mentioned above, there is an obvious subdivision of $\mathscr{H}$ into three disjoint sublcasses, $\mathscr{M}_{1}, \mathscr{H}_{2}^{1}, \mathscr{M}_{2}^{2}$. Typical examples of groups in the three classes are, respectively: infinite simple groups and quasicyclic groups, infinite non-simple $\mathscr{D}_{2}$-groups, countable direct powers of finite simple groups. Some discussion concerning the structure of $\mathscr{M}$-groups (particularly $\mathscr{M}_{2}^{1} \cup \mathscr{K}_{2}^{2}$-groups) is given. The minimal abelian groups are determined.

In Section 3, attention is given to abelian groups of finite height. Necessary and sufficient conditions are found under which groups of any one of the following types has finite height: divisible groups, direct sums of cyclic groups, torsion groups.

In Pride (to appear), an example was given showing that poly-minimal groups need not have finite height. Other examples show that not every group of finite height is poly-minimal. For instance, if $G$ is the direct product of $\aleph_{1}$ copies of a finite simple group $S$ (where $S \neq 1$ ) then $G$ has height 2. For if $H \prec G$ then $H$ is equally as large as the direct product of $\aleph_{0}$ copies of $S$ (use Robinson (1972), Theorem 5.45). It is not difficult to show that $G$ is not poly-minimal. It can thus be seen that the minimal groups do not constitute the 'building blocks' for groups of finite height. 
Nevertheless, there is a connection between minimal groups and height. Suppose $G$ is a group and $M_{1}, \ldots, M_{n}$ are minimal groups, no two of which are equally large, and $M_{i} \preccurlyeq G$ for $i=1, \ldots, n$. Then it is shown in Section 4 that $M_{1} \times \ldots \times M_{n} \preccurlyeq G$ and that $G$ has height at least $n$. It follows that if there are infinitely many minimal group $M \prec G$, no two of which are equally large, then $G$ does not satisfy Max- $\preccurlyeq$.

The fact that not every poly-minimal group has finite height means that there is interest in looking for classes $\mathscr{Y}$ of minimal groups such that every poly- $\mathscr{Y}$ group does have finite height. One suitable choice for $\mathscr{Y}$ is the class of quasicyclic groups. Indeed every poly-(quasicyclic or finite) group has a subgroup of finite index which is the direct product of finitely many quasicyclic groups (see Robinson (1972), p. 69). It thus follows (using Pride (to appear), Theorem 4.5) that every poly-(quasicyclic or finite) group has finite height.

Another suitable choice for $\mathscr{Y}$ is the class of infinite cyclic groups. It is not difficult to show that every poly-(cyclic or finite) group has finite height. More generally one can take $\mathscr{Y}$ to be the class of infinite $\mathscr{D}_{2}$-groups. In Section 5 attention is given to the class of poly- $\mathscr{D}_{2}$ groups. This class is quite extensive. It includes polycyclic groups, groups with a (finite) composition series, and $\mathscr{D}_{1}$-groups satisfying Max-sn (see Wilson (1971)). By introducing a rank function (which is a generalization of the Hirsch number for polycyclic groups) it is shown that every poly- $\mathscr{D}_{2}$ group has finite height.

Unless otherwise stated, notation will be as in Pride (to appear). In order to avoid annoying distinctions between finite and infinite groups, it is convenient to extend the definitions of $\simeq$ and $\preccurlyeq$ given in Pride (to appear). Thus any two finite groups are defined to be equally large, and if $H$ is finite and $G$ infinite then $G$ is defined to be strictly larger than $H$.

For Theorems 3-6 the reader is assumed to be familiar with the basic theory of abelian groups (see Rotman (1973), Chapter 9 for example). Notation for abelian groups will be as in Rotman (1973), except that $\sigma\left(p^{\infty}\right)$ groups will be called quasicyclic, the additive group of the rationals will be denoted by $\mathbf{Q}$, and the cyclic group of order $n$ will be denoted by $\mathbf{Z}_{n}$.

The class of abelian groups will be denoted by $\mathfrak{A}$, and $\mathfrak{H}$ will denote the class of groups $G$ with the following property: for every integer $n>0, G$ has only finitely many distinct subgroups of index $n$.

\section{On minimal groups}

The aim of this section is to obtain some information on the structure of minimal groups. In this connection it is convenient to divide $\mathscr{M}$ into three disjoint subclasses as follows. Let $\mathscr{M}_{1}$ be the class of minimal groups $G$ with the property that the 
intersection of all subgroups of finite index in $G$ again has finite index in $G$. Let $\mathscr{H}_{2}=\mathscr{M} \backslash \mathscr{M}_{1}$. Subdivide $\mathscr{M}_{2}$ as follows: $\mathscr{M}_{2}^{1}=\mathscr{H}_{2} \cap \mathfrak{H}$ and $\mathscr{M}_{2}^{2}=\mathscr{M}_{2} \mid \mathscr{K}_{2}^{1}$.

The class $\mathscr{K}_{1}$ is enormous, containing for example all infinite simple groups. In particular $\mathscr{M}_{1}$ contains groups of arbitrarily large cardinality. On the other hand:

THEOREM 1. Every $\mathscr{M}_{2}$-group has cardinality at most $2^{\mathrm{N}_{0}}$. This bound can be attained.

Proof. Let $G$ be an $\mathscr{M}_{2}$-group and let $N_{i}(i=1,2, \ldots)$ be distinct normal subgroups of $G$ with $\left|G: N_{i}\right|<\infty$. Let $N=\bigcap_{i=1}^{\infty} N_{i}$. Then $G / N \simeq G$, and $G / N$ can be embedded in

$$
\prod_{i=1}^{\infty} \frac{G}{N_{i}}
$$

Thus $G / N$, and therefore $G$, has cardinality at most $2^{N_{0}}$.

The $\mathscr{H}_{2}$-group discussed in Wilson (1971), p. 380, has cardinality $2^{N_{0}}$. This proves the theorem.

The class $\mathscr{M}_{2}^{1}$ contains all minimal just-infinite groups which are not simple-byfinite (by Wilson (1970), Theorem A). It seems reasonable to suggest that $\mathscr{H}_{2}^{1}$ consists only of those groups which are equally as large as some minimal justinfinite group.

The next result gives a reasonable amount of information concerning the structure of $\mathscr{M}_{2}^{2}$-groups.

THEOREM 2. If $G$ is an $\mathscr{M}_{2}^{2}$-group then Ghas a subgroup of finite index which is locally a finite direct power of some finite simple group $S$.

Proof. Since $G$ does not lie in $\mathfrak{H}$ it follows from Wilson (1970), Lemma 1, that $G$ has a subgroup $G_{0}$ of finite index with infinitely many distinct subgroups $K_{i} \triangleleft G_{0}$ $(i \in I)$, where $G_{0} / K_{i}$ is isomorphic to a finite simple group $S$. Let $K=\bigcap_{i \in I} K_{i}$, and consider $G_{0}=G_{0} / K$. Note that $G_{0} \simeq G$. Note also that $G_{0}$ belongs to the variety $\mathfrak{U}$ generated by $S$.

Assume first that $S$ is non-abelian. Let $\mathfrak{B}$ be the variety generated by all proper subgroups of $S$; then $S$ does not belong to $\mathfrak{B}$ (Neumann (1967), 51.34). Let $W\left(G_{0}\right)$ be the verbal subgroup of $\bar{G}_{0}$ corresponding to the variety $\mathfrak{B}$. If $\left|G_{0}: W\left(G_{0}\right)\right|=\infty$, consider $\bar{G}_{0} / W\left(\bar{G}_{0}\right)$, which is equally as large as $\bar{G}_{0}$ and belongs to $\mathfrak{B}$. This must have a subgroup of finite index which can be mapped onto a normal subgroup $U$ of finite index in $\bar{G}_{0}$, and clearly $U \in \mathfrak{B}$. Now there exists $K_{i}$ such that $U \$ R_{i}$ (where $K_{i}=K_{i} / K$ ). Then $U / U \cap R_{i}$ is isomorphic to $S$. But this implies that $S \in \mathfrak{B}$, a contradiction. It now follows $\left|G_{0}: W\left(G_{0}\right)\right|<\infty$, and so $G \simeq W\left(G_{0}\right)$. Thus $W\left(G_{0}\right)$ 
has a normal subgroup of finite index which can be mapped onto a subgroup $L$ of finite index in $G$. Since $W\left(G_{0}\right)$ is locally a finite direct power of $S$ (Wilson (1970), Lemma 7 '), and since the property 'being locally a finite direct power of $S$ ' is closed under taking normal subgroups and homomorphic images, it follows that $L$ is locally a finite direct power of $S$.

Now suppose that $S$ is abelian, so that $G_{0}$ is an elementary abelian group. By Prüfer's Theorem, $G_{0}$ is the direct sum of $p$-cycles for some prime $p$. Moreover, the number of summands must be countable, for otherwise $G_{0}$ would be uncountable and yet would have a countable homomorphic image $H$, so that $H \prec \bar{G}_{0}$. Since the direct sum of countably many $p$-cycles is easily seen to be minimal, this completes the proof.

Some comments are in order. (In the following discussion $S$ will denote a non-trivial finite simple group.)

It can be shown without too much difficulty that the direct product $\mathrm{P}(S)$ of countably many copies of $S$ is minimal (for the proof when $S$ is non-abelian see Pride (to appear), Example 4.3). If $S$ is abelian then any group which is locally a finite direct power of $S$ is the direct product of copies of $S$ (by Prüfer's Theorem), and so in this case the only minimal group which is locally a finite direct power of $S$ is (isomorphic to) $\mathrm{P}(S)$. On the other hand, if $S$ is non-abelian it is conceivable that there are minimal groups which are locally a finite direct power of $S$, but which are not equally as large as $\mathrm{P}(S)$. However, I have not been able to find any such groups. It seems hopeful that for suitable choice of ring $R$, the Boolean power $\operatorname{BP}(S, R)$ (as defined in Neumann and Yamamuro (1965)) should be minimal, but except for the case when $R$ is the ring $\mathscr{F}$ of finite-cofinite subsets of a countable set, I have not been able to show this. Notice that $\operatorname{BP}(S, \mathscr{F})$ has a subgroup of finite index isomorphic to $\mathrm{P}(S)$.

The last result of this section gives a complete description of the abelian $\mathscr{M}$ groups. This obviously enables one to describe the soluble $\mathscr{M}$-groups, for it is clear that every soluble $\mathscr{M}$-group has an abelian $\mathscr{M}$-subgroup of finite index.

THEOREM 3. (i) $\mathfrak{A} \cap \mathscr{H}_{1}$ is the class of groups $Q \oplus B$, where $Q$ is quasicyclic and $B$ is a finite abelian group.

(ii) $\mathfrak{U} \cap \mathscr{M}_{2}^{1}$ is the class of groups $C \oplus B$, where $C$ is infinite cyclic and $B$ is a finite abelian group.

(iii) $\mathfrak{A} \cap \mathscr{H}_{2}^{2}$ is the class of direct sums of countably many finite cycles, almost all of which are p-cycles for a fixed prime $p$.

Proof. First consider (i). It is easily shown that any finite extension of a quasicyclic group lies in $\mathscr{H}_{1}$. On the other hand, suppose $G$ lies in $\mathfrak{A} \cap \mathscr{M}_{1}$. Then $G$ has a subgroup $Q$ of finite index which has no proper subgroups of finite index. Thus $Q$ 
is divisible, and so $G=Q \oplus B$ where $B$ is finite. Moreover, $Q$ has a quasicyclic homomorphic image $\bar{Q}$. Since $Q$ is minimal $Q \preccurlyeq \bar{Q}$, and so $Q$ and $\bar{Q}$ are isomorphic.

The fact that $\mathfrak{A} \cap \mathscr{H}_{2}^{2}$ consists precisely of the groups described in (iii) follows from the proof of Thoerem 2 (see also the comments following the proof).

In order to deal with (ii) it is necessary to show that no abelian torsion group lies in $\mathscr{H}_{2}^{1}$. Suppose $H$ is a minimal abelian torsion group, and let $D$ be a basic subgroup of $H$. Then $H / D$ is divisible; so either $H=D$ or $H / D \simeq H$. It follows from what has already been shown that in the former case $H$ lies in $\mathscr{A}_{2}^{2}$, while in the latter case $H$ lies in $\mathscr{M}_{1}$.

It is easy to show that any finite extension of an infinite cyclic group lies in $\mathscr{M}_{2}^{1}$. On the other hand, suppose $G$ lies in $\mathfrak{A} \cap \mathscr{M}_{2}^{1}$. It follows from the previous paragraph that the quotient group $K$ of $G$ by its torsion subgroup must be infinite. So $K \simeq G$. Let $x$ be a non-trivial element of $K$ and define a subset $L$ of $K$ to be independent of $x$ if an equation $\gamma x+\lambda_{1} y_{1}+\ldots+\lambda_{n} y_{n}=0\left(n \geqslant 0, \gamma, \lambda_{1}, \ldots, \lambda_{n}\right.$ integers, $y_{1}, \ldots, y_{n} \in L$ ) implies $\gamma=0$. Let $E$ be a maximal set independent of $x$ (which exists by Zorn's Lemma). Then $\operatorname{sgp}\{x, E\}=\operatorname{sgp}\{x\} \oplus \operatorname{sgp} E$. Moreover, the maximality of $E$ implies that $K / \operatorname{sgp}\{x, E\}$ is a torsion group, and so it follows from the previous paragraph that $|K: \operatorname{sgp}\{x, E\}|<\infty$. Consequently $G \simeq \operatorname{sgp}\{x, E\}$. But $\operatorname{sgp}\{x\} \preccurlyeq \operatorname{sgp}\{x, E\}$, so $G \simeq \operatorname{sgp}\{x\}$. It now follows easily that $G$ is the direct sum of an infinite cyclic group and a finite group.

This completes the proof of Theorem 3.

\section{Abelian groups of finite height}

In this section only, group will mean abelian group.

THEOREM 4. A divisible group has finite height if and only if it is isomorphic to the direct sum of $n$ quasicyclic groups for some non-negative integer $n$, in which case the group has height exactly $n$.

PROOF. Let $B=\Sigma_{1}^{r} Q_{i}$, where each $Q_{i}$ is quasicyclic, and let $C$ be a homomorphic image of $B$. Then it is not difficult to show that $C$ is isomorphic to $\sum_{i \in I} Q_{i}$ for some subset $I$ of $\{1, \ldots, r\}$. Moreover, if $I$ is properly contained in $\{1, \ldots, r\}$ then there is no homomorphism of $C$ back onto $B$.

Now suppose $G=\sum_{1}^{n} D_{i}$ where each $D_{i}$ is quasicyclic. Since divisible groups have no proper subgroups of finite index, it follows from the previous paragraph that $H \preccurlyeq G$ if and only if $H \simeq \sum_{i \in I} D_{i}$ for some subset $I$ of $\{1, \ldots, n\}$. It also follows from the previous paragraph that if $J$ is a proper subset of $I$ then $\sum_{j_{\in} J} D_{j} \prec \sum_{i \in I} D_{i}$. Consequently $G$ has height exactly $n$. 
Conversely, suppose $G$ is a divisible group of finite height. Now every divisible group is isomorphic to the direct sum of quasicyclic groups and copies of $\mathbf{Q}$. But for every integer $r \geqslant 0, Q$ can be mapped onto the direct sum of $r$ quasicyclic groups. Consequently, $\mathbf{Q}$ has infinite height by the previous paragraph and so $\mathbf{Q}$ cannot occur as a direct summand of $G$. Thus $G$ is isomorphic to the direct sum of quasicyclic groups, and this direct sum can have only finitely many summands, again by the previous paragraph.

This completes the proof.

THEOREM 5. Let $G$ be a direct sum of cyclic groups. Then $G$ has finite height if and only if:

(i) the rank of $G / t G$ is finite;

(ii) $t G$ has bounded order;

(iii) $|G|<\aleph_{\omega}$ where $\omega$ is the least infinite ordinal.

(Recall that a group has bounded order if there is an integer $n$ such that $n g=0$ for all elements of the group.)

Proof. First suppose that $G$ has finite height.

Since a free abelian group of rank $n$ has height $n$ (Pride (to appear), Example 4.1) it is clear that (i) holds.

To show that (ii) holds, it suffices to show that $(t G)_{p}$ has bounded order for all primes $p$, and that for almost all primes $(t G)_{p}=0$. Suppose first, by way of contradiction, that for an infinite set $\pi$ of primes $(t G)_{p} \neq 0$ for $p \in \pi$. Let $\pi_{1}, \pi_{2}, \ldots$ be a sequence of subsets of $\pi$ with $\pi_{i} \supset \pi_{i+1}$ and $\pi_{i} \backslash \pi_{i+1}$ infinite for $i=1,2, \ldots$ Now obviously $t G$ (and thus $G$ ) can be mapped onto the direct sum $A_{i}$ of cycles of order $p$ with $p$ ranging over $\pi_{i}$. It is not difficult to show that $A_{i+1} \prec A_{i}$, so $t G$ does not have finite height. Next suppose, again by way of contradiction, that for some prime $p,(t G)_{p}$ has unbounded order. Then $t G$ (and hence $G$ ) can be mapped onto a countable direct sum $D_{n}$ of cycles of order $p^{n}$ for arbitrarily large $n$. Now it is not difficult to show that $D_{0} \prec D_{1} \prec \ldots \prec D_{n}$, and so $C$ has height at least $n$. Since $n$ can be chosen arbitrarily large, this means that $G$ has infinite height-a contradiction.

It remains to show that (iii) holds. Suppose $|G|=\kappa$. If $\aleph_{0} \leqslant \alpha \leqslant \kappa$ then $G$ has a homomorphic image $G_{\alpha}$ of cardinality $\alpha$. Clearly if $\aleph_{0} \leqslant \alpha<\beta \leqslant \kappa$ then $G_{\alpha}<G_{\beta}$. Thus $\kappa<\boldsymbol{\aleph}_{\omega}$.

Now suppose that $G$ satisfies (i)-(iii). In order to show that $G$ has finite height some preliminary discussion is required. 
By a signature will be meant a sequence

$$
\sigma=\left(m, n, \mathbf{s}_{1}, \mathbf{s}_{2}, \ldots\right) \text {, }
$$

where $m, n$ are non-negative integers and where for $i=1,2, \ldots, s_{i}$ is an $(n+1)$-tuple $\left(s_{i 0}, \ldots, s_{i n}\right)$ of non-negative integers with either $s_{i j}=0$ or $s_{i j}>s_{i(j+1)}$. In addition, almost all of the $s_{i}$ are required to be 0 . If $\tau=\left(k, l, \mathbf{t}_{1}, \mathbf{t}_{2}, \ldots\right)$ is another signature then write $\tau \leqslant \sigma$ if $k \leqslant m, l \leqslant n, \mathbf{t}_{i} \leqslant \mathbf{s}_{i}$ for $i=1,2, \ldots$. Here, if $\mathbf{t}=\left(t_{0}, \ldots, t_{l}\right)$ and $\mathbf{s}=\left(s_{0}, \ldots, s_{n}\right)$ are respectively an $(l+1)$-tuple and an $(n+1)$-tuple of integers where $l \leqslant n$, then $\mathbf{t} \leqslant \mathrm{s}$ means $t_{0} \leqslant s_{0}, \ldots, t_{i} \leqslant s_{l}$.

A group $B$ satisfying (i)-(iii) determines a signature as follows: the first coordinate is the rank of $B / t B$; if $|B|=\aleph_{n}$ then the second coordinate is $n$, and if $B$ is finite the second coordinate is zero. Let $p_{1}, p_{2}, \ldots$ be the sequence of primes. For $i=1,2, \ldots$ let

$$
s_{i n}=\max \left\{r: \text { there are } \aleph_{n} \text { summands of } B \text { of order } p_{i}^{r}\right\}
$$

and for $0 \leqslant j<n$,

$$
s_{i j}=\max \left\{r: r>s_{i(j+1)} \text {, there are } \aleph_{j} \text { summands of } B \text { of order } p_{i}^{r}\right\}
$$

(Adopt the convention $\max \phi=0$.) Then put $\mathbf{s}_{i}=\left(s_{i 0}, \ldots, s_{i n}\right)$.

It is not too difficult to establish the following three facts.

(a) Given a signature $\sigma$ as in $\left(^{*}\right)$, there is a group [ $\left.\sigma\right]$ whose signature is precisely $\sigma$, defined as follows. Let $U$ be a free abelian group of rank $m$. For $i=1,2, \ldots$ let $V_{i}$ be the direct sum of $\boldsymbol{\aleph}_{n}$ cycles of order $p_{i}^{s_{i}}, \boldsymbol{\aleph}_{n-1}$ cycles of order $p_{i}^{s_{i}(n-1)}, \ldots, \boldsymbol{\aleph}_{0}$ cycles of order $p_{i}^{s i 0}$. Then $[\sigma]=U \oplus \sum_{1}^{\infty} V_{i}$.

(b) If $B$ satisfies (i)-(iii) and has signature $\sigma$ then $B \simeq[\sigma]$.

(c) If $B$ satisfies (i)-(iii) and has signature $\sigma$ and if $C \preccurlyeq B$ then $C$ is equally as large as a group $B_{1}$ satisfying (i)-(iii), where the signature $\tau$ of $B_{1}$ is such that $\tau \leqslant \sigma$.

It now follows easily from (b) and (c) that if $H \preccurlyeq G$ then $H \simeq[\tau]$ for some $\tau$ with $\tau \leqslant \sigma$. Since the number of signatures $\tau$ with $\tau \leqslant \sigma$ is finite, it follows that $G$ has finite height.

This proves Theorem 5 .

REMARK. It can be seen from the proofs of Theorems 4 and 5 that if $G$ is either a divisible group or a direct sum of cyclic groups, and if $G$ has finite height, then up to equivalence under $\simeq$, there are only finitely many groups $H$ with $H \preccurlyeq G$. This fact will be needed for the proof of Theorem 6. (Some further comments related to this remark will be made at the end of Section 4.) 
Theorem 6. Let $G$ be a torsion group, and let $B$ be a basic subgroup of $G$. Then $G$ has finite height if and only if:

(i) $G / B$ is the direct sum of finitely many quasicyclic groups;

(ii) $B$ is of bounded order;

(iii) $|G|<\aleph_{\omega}$ where $\omega$ is the least infinite ordinal.

Proof. First suppose that $G$ has finite height.

Since $G / B \preccurlyeq G, G / B$ has finite height, so (i) holds by Theorem 4 .

To show that (ii) holds it suffices to show that $B_{p}$ has bounded order for all primes $p$, and that for almost all primes $B_{p}=0$. Suppose first, by way of contradiction, that for an infinite set $\pi$ of primes $B_{p} \neq 0$ for $p \in \pi$. Since $G / B$ is the direct sum of finitely many quasicyclic groups there is an infinite subset $\pi^{\prime}$ of $\pi$ such that $\Sigma_{p \in \pi^{\prime}} B_{p}$ is a direct summand of $G$. But by Theorem $5, \Sigma_{p \in \pi^{\prime}} B_{p}$ has infinite height, and so therefore does $G$, contrary to assumption. Next suppose, again by way of contradiction, that for some prime $p, B_{p}$ has unbounded order. Then for arbitrarily large $n$ there is a factor group $B / N$ of $B$ isomorphic to the direct sum of $\aleph_{0}$ cycles of order $p^{n}$. Since $B / N$ is of bounded order and is pure in $G / N$, it is a direct summand, so $G$ has $B / N$ as a homomorphic image. But $B / N$ has height $n$, and since $n$ can be chosen arbitrarily large, this implies that $G$ has infinite height-a contradiction.

Now (ii) implies that $B$ is a direct summand, and so (iii) holds using Theorem 5(iii).

Next suppose that $G$ satisfies (i)-(iii). Then $B$ is a direct summand, so $G$ can be written as $B \oplus C$, where $C$ is the direct sum of finitely many quasicyclic groups. Suppose $L \preccurlyeq G$. Then there is a subgroup $H$ of finite index in $B \oplus C$ which can be mapped onto a subgroup $K$ of finite index in $L$. Obviously $H=B_{1} \oplus C$ for some subgroup $B_{1}$ of finite index in $B$. Let $D$ be the image of $C$ under the mapping $H \rightarrow K$. Since $D$ is divisible it is a direct summand, so $K=U \oplus D$ where $U$ is a homomorphic image of $B_{1}$. Hence $U \preccurlyeq B, D \preccurlyeq C$. Since (up to equivalence under $\simeq$ ) there are only finitely many group $U \preccurlyeq B, D \preccurlyeq C$ (see remark above), it follows that $G$ has finite height.

This completes the proof.

\section{Direct products of minimal groups}

The principal aim of this section is to show how the height of a group $G$ is influenced by the minimal groups $M$ with $M \preccurlyeq G$. In the course of looking at this question one is led to consider direct products of minimal groups, and so the section begins with a discussion of this subject. 
It was shown in Pride (to appear), Theorem 4.5, that the direct product of $n$ minimal groups has height at most $n$. It turns out that if no two of the direct factors are equally large then such a direct product has height exactly $n$.

THEOREM 7. Let $M_{1}, \ldots, M_{n}$ be minimal groups, no two of which are equally large. Then:

(i) $M_{1} \prec M_{1} \times M_{2} \prec \ldots \prec M_{1} \times \ldots \times M_{n}$;

(ii) $M_{1} \times \ldots \times M_{n}$ has height $n$.

Proof. The proof of (i) is by induction on $n$. If $n=1$ the result is trivial.

Assume $n>1$, and suppose by way of contradiction that

$$
M_{1} \times \ldots \times M_{n-1} \simeq M_{1} \times \ldots \times M_{n} .
$$

Now it follows from Pride (to appear), Theorem 4.5 and the inductive hypothesis that if $M$ is a minimal group with $M \preccurlyeq M_{1} \times \ldots \times M_{n-1}$ then $M \simeq M_{i}$ for some $i$ with $1 \leqslant i \leqslant n-1$. But $M_{n} \preccurlyeq M_{1} \times \ldots \times M_{n-1}$ and so there exists $i(1 \leqslant i \leqslant n-1)$ such that $M_{n} \simeq M_{i}$-a contradiction.

It is now obvious that (ii) holds, using (i) and Pride (to appear), Theorem 4.5.

When one comes to consider direct powers of minimal groups it is found that various things can happen. It has already been pointed out in Pride (to appear) that if $B$ is a minimal just-infinite group not lying in $\mathscr{D}_{2}$ then $B \times B \simeq B$. In fact the work in Pride (to appear), Sections 4.2 and 4.3, shows the following. Let $G$ be a minimal justinfinite group. If $G$ lies in $\mathscr{D}_{2}$ then $G^{n}$ has height $n$, while if $G$ does not lie in $\mathscr{D}_{2}$ then $G^{n} \simeq G$. The group $\mathrm{P}(S)$ of Section 2 has the property that the direct product of $\aleph_{0}$ copies of $\mathrm{P}(S)$ is equally as large as $\mathrm{P}(S)$.

The next theorem is the main result of this section.

THEOREM 8. Let $M_{1}, \ldots, M_{n}$ be minimal groups, no two of which are equally large. Suppose $G$ is a group with $M_{i} \preccurlyeq G$ for $i=1, \ldots, n$. Then $M_{1} \times \ldots \times M_{n} \preccurlyeq G$, and $G$ has height at least $n$.

Proof. The proof is by induction on $n$. If $n=1$ the result is trivial.

Suppose $n>1$. By the inductive hypothesis, $G$ has subgroups $G_{0}, N_{1}, N_{2}$ with $\left|G: G_{0}\right|<\infty, N_{1}, N_{2} \triangleleft G_{0}, G_{0} / N_{1}$ isomorphic to a subgroup of finite index in $M_{1} \times \ldots \times M_{n-1}, G_{0} / N_{2}$ isomorphic to a subgroup of finite index in $M_{n}$. Now $\left|G_{0}: N_{1} N_{2}\right|<\infty$. For suppose $\left|G_{0}: N_{1} N_{2}\right|=\infty$. Then $G_{0} /\left(N_{1} N_{2}\right) \simeq M_{n}$ and $G_{0} /\left(N_{1} N_{2}\right) \preccurlyeq M_{1} \times \ldots \times M_{n-1}$. Thus, by Theorem $7, M_{n} \simeq M_{i}$ for some $i$ with $1 \leqslant i \leqslant n-1$. This is a contradiction.

Since $\left|G_{0}: N_{1} N_{2}\right|<\infty, G \simeq N_{1} N_{2}$. Thus $\left(N_{1} N_{2}\right) / N_{1} \cap N_{2} \preccurlyeq G$. But $\left(N_{1} N_{2}\right) / N_{1} \cap N_{2}$ is isomorphic to $\left(N_{1} N_{2}\right) / N_{1} \times\left(N_{1} N_{2}\right) / N_{2}$. Moreover, $\left(N_{1} N_{2}\right) / N_{1} \simeq M_{1} \times \ldots \times M_{n-1}$ and $\left(N_{1} N_{2}\right) / N_{2} \simeq M_{n}$. So $\left(N_{1} N_{2}\right) / N_{1} \cap N_{2} \simeq M_{1} \times \ldots \times M_{n} \preccurlyeq G$, as required. 
The fact that $G$ has height at least $n$ follows from Theorem 7 .

This completes the proof.

COROLlaRY. Suppose $M_{i}(i=1,2, \ldots)$ are minimal groups, no two of which are equally large. If $G$ is a group with $M_{i} \prec G$ for $i=1,2, \ldots$ then $G$ does not satisfy Max- $\leqslant$.

For $M_{1} \prec M_{1} \times M_{2} \prec M_{1} \times M_{2} \times M_{3} \prec \ldots \prec G$, by Theorems 7 and 8 .

It should be remarked that if $G$ is such that there are only finitely many distinct (up to $\simeq$ ) minimal groups $M$ with $M \preccurlyeq G$, then $G$ may satisfy Max- $\preccurlyeq$ (for example, if $G$ is minimal), but on the other hand it need not (for example, if $G$ is the group constructed in Pride (to appear), Theorem 4.4.).

It follows from Theorem 8 that if $G$ is a group of finite height then there are only finitely many distinct (up to $\simeq$ ) minimal groups $M$ with $M \preccurlyeq G$. It is not in general true that if $G$ is a group of finite height then there are only finitely many distinct (up to $\simeq$ ) groups $H$ (not necessarily minimal) with $H \preccurlyeq G$. Examples showing this have been found by $A$. Hales (1979).

\section{Poly- $\mathscr{D}_{2}$ groups}

The aim of this section is to establish the following result.

THEOREM 9. Every poly- $\mathscr{D}_{2}$ group has finite height.

In order to prove this theorem some preliminary discussion is needed. Throughout this section the following result will be used without explicit mention: every subgroup of finite index in a $\mathscr{D}_{2}$-group is again a $\mathscr{D}_{2}$-group (Tretkoff (1976)).

Let $G$ be a poly- $\mathscr{D}_{2}$ group. By a $\mathscr{D}_{2}$-series for $G$ will be meant a series

$$
\mathscr{S}: 1=G_{0} \unlhd G_{1} \unlhd \ldots \unlhd G_{n}=G,
$$

where $G_{i+1} / G_{i}$ is a $\mathscr{D}_{2}$-group for $i=0, \ldots, n-1$. Using Schreier's Theorem, it is not difficult to establish that any two $\mathscr{D}_{2}$-series for $G$ have the same number of infinite factors. Denote this number by $\rho_{i}(G)$.

Now consider a $\mathscr{D}_{2}$-series $\mathscr{S}$ for $G$ as above, and suppose $G_{k+1} / G_{k}$ is the last infinite factor. Let $\rho_{f}(\mathscr{S})$ be the sum of the orders of the finite factors in the $\mathscr{D}_{2}$ series $G_{0} \unlhd G_{1} \unlhd \ldots \unlhd G_{k}$ (if $k$ does not exist put $\left.\rho_{f}(\mathscr{S})=0\right)$. Denote $\min \left\{\rho_{f}(\mathscr{S}): \mathscr{S}\right.$ is a $\mathscr{D}_{2}$-series for $\left.G\right\}$ by $\rho_{f}(G)$. A $\mathscr{D}_{2}$-series $\mathscr{S}$ for $G$ with $\rho_{f}(G)=\rho_{f}(\mathscr{S})$ will be called a minimal series. Clearly $\rho_{f}(G)$ is an invariant of $G$.

The rank of $G$, denoted $\rho(G)$, will be the ordered pair $\left(\rho_{i}(G), \rho_{f}(G)\right)$. 
If $\left(m_{1}, m_{2}\right),\left(n_{1}, n_{2}\right)$ are two ordered pairs of integers then write $\left(m_{1}, m_{2}\right)<\left(n_{1}, n_{2}\right)$ if either $m_{1}<n_{1}$ or $m_{1}=n_{1}$ and $m_{2}<n_{2}$ (the lexicographical ordering).

It is well-known that for every polycyclic group $G$ (and more generally for every poly-(cyclic or finite) group $\rho_{f}(G)=0$. Thus $\rho(G)$ can be identified with $\rho_{i}(G)$. This number is usually called the Hirsch number. On the other hand, if $G=\operatorname{SL}(n, F)$ where $F$ is an infinite field having a finite number, say $m$, of $n$th roots of unity then $\rho(G)=(1, m)$, while if $G=\operatorname{Sym}\left(\boldsymbol{\aleph}_{n}\right)$ then $\rho(G)=(n+2,2)(\operatorname{see} \operatorname{Scott}(1964), 11.3 .4)$.

LeMma. Let $G$ be a poly- $\mathscr{D}_{2}$ group.

(i) If $B$ is a subgroup of finite index in $G$ then $B$ is a poly- $\mathscr{D}_{2}$ group and $\rho_{i}(B)=\rho_{i}(G), \rho_{f}(B) \leqslant \rho_{f}(G)$.

(ii) If $C$ is a homomorphic image of $G$ then $C$ is a poly $\mathscr{D}_{2}$ group and $\rho(C) \leqslant \rho(G)$. Moreover, $\rho(C)=\rho(G)$ implies $C \simeq G$.

Proof. Let $\mathscr{S}: 1=G_{0} \triangleleft G_{1} \triangleleft \ldots \triangleleft G_{n}=G$ be a minimal series for $G$.

To prove (i), let $B_{i}=B \cap G_{i}$ for $i=0, \ldots, n$. Then $B_{i+1} / B_{i}$ is isomorphic to a subgroup of finite index in $G_{i+1} / G_{i}(i=0, \ldots, n-1)$. Thus

$$
\mathscr{T}: 1=B_{0} \unlhd B_{1} \unlhd \ldots \unlhd B_{n}=B
$$

is a $\mathscr{D}_{2}$-series for $B$. Moreover, $\mathscr{T}$ has the same number of infinite factors as $\mathscr{S}$ and so $\rho_{i}(B)=\rho_{i}(G)$. The finite factors of $\mathscr{T}$ have orders not exceeding the orders of the corresponding finite factors of $\mathscr{S}$. Thus $\rho_{f}(B) \leqslant \rho_{f}(\mathscr{T}) \leqslant \rho_{f}(\mathscr{S})=\rho_{f}(G)$.

To prove (ii), let $\theta$ be the homomorphism of $G$ onto $C$, and for $i=0, \ldots, n$ let $C_{i}=\theta\left(G_{i}\right)$. Then $\theta_{i}: G_{i+1} / G_{i} \rightarrow C_{i+1} / C_{i}$ defined by $\theta_{i}\left(G_{i} x\right)=C_{i} \theta(x)$ is an epimorphism. This implies in particular that $\mathscr{U}: 1=C_{0} \unlhd C_{1} \unlhd \ldots \unlhd C_{n}=C$ is a $\mathscr{D}_{2^{-}}$ series for $C$. If one of the infinite factors of $\mathscr{S}$ gets mapped (by the appropriate $\theta_{j}$ ) to a finite factor then $\rho_{i}(C)<\rho_{i}(G)$. Otherwise all the infinite factors are mapped isomorphically, and the finite factors of $\mathscr{S}$ are mapped homomorphically onto the finite factors of $\mathscr{U}$. Thus $\rho_{i}(C)=\rho_{i}(G)$ and $\rho_{f}(C) \leqslant \rho_{f}(\mathscr{U}) \leqslant \rho_{f}(\mathscr{S})=\rho_{f}(G)$.

Now suppose $\rho(C)=\rho(G)$. This means that if $G_{k+1} / G_{k}$ is the last infinite factor of $\mathscr{S}$ then for $0 \leqslant i \leqslant k, \theta_{i}$ is an isomorphism. Consequently, the restriction of $\theta$ to $G_{k+1}$ is an isomorphism, and so $G$ and $C$ both have subgroups of finite index isomorphic to $G_{k+1}$. Thus $G \simeq C$.

Proof of Theorem 9. It is clearly sufficient to establish that if $G$ is a poly- $\mathscr{D}_{2}$ group and $H \prec G$ then $H$ is equally as large as a poly- $\mathscr{D}_{2}$ group $L$ with $\rho(L)<\rho(G)$. 
Now if $H \prec G$ then $G$ has a subgroup $K$ of finite index which can be mapped onto a subgroup $L$ of finite index in $H$. By the lemma, $\rho(K) \leqslant \rho(G)$. Moreover, since $L \prec K, \rho(L)<\rho(K)$, again by the lemma. This completes the proof.

It has already been noted that if $G$ is poly-(cyclic or finite) then $\rho(G)$ is essentially the Hirsch number of $G$, and it follows easily from the proof of Theorem 9 that every poly-(cyclic or finite) group has height less than or equal to its Hirsch number. It can be shown that the height is equal to the Hirsch number if and only if the group is nilpotent-by-finite.

One has the feeling that finitely generated nilpotent-by-finite groups are somehow 'smaller' than polycyclic groups in general, and there are results in the literature to support this notion (for example, Wolf (1968)). The reader might be tempted to think that the results mentioned in the previous paragraph give support to the opposite viewpoint. However, this is purely illusionary, for obviously two groups of different height need not be comparable. It is in fact easy to see that no nilpotentby-finite group can be larger than any group which is not nilpotent-by-finite.

\section{References}

B. Baumslag and S. J. Pride (1978), 'Groups with two more generators than relators', J. London Math. Soc., (2) 17, 425-426.

A. Hales (1979), 'Groups of height four', preprint

B. H. Neumann and S. Yamamuro (1965), 'Boolean powers of simple groups', J. Austral. Math. Soc. 5, 315-324.

H. Neumann (1967), Varieties of groups (Ergebnisse der Mathematik, Bd 37, Springer-Verlag, Berlin, Heidelberg, New York).

S. J. Pride (to appear) 'The concept of "largeness" in group theory', Proc, Conf. on Word and Decision Problems in Algebra and Group Theory (Oxford).

D. J. S. Robinson (1972), Finiteness conditions and generalized soluble groups Vol. 1 (Ergebnisse der Mathematik, Bd 62, Springer-Verlag, Berlin, Heidelberg, New York).

J. J. Rotman (1973), The theory of groups: an introduction (2nd edition, Allyn and Bacon, Boston).

W. R. Scott (1964), Group theory (Prentice-Hall, Englewood Cliffs, New Jersey).

C. Tretkoff (1976), 'Some remarks on just-infinite groups', Comm. Algebra 4, 483-489.

J. S. Wilson (1970), 'Groups satisfying the maximal condition for normal subgroups', Math. $Z$. 118, $107-114$.

J. S. Wilson (1971), 'Groups with every proper quotient finite', Proc. Camb. Phil. Soc. 69, 373-391.

J. A. Wolf (1968), 'Growth of finitely generated solvable groups and curvature of Riemannian manifolds', $J$. Differential Geometry 2, 421-446.

Faculty of Mathematics

Open University

Milton Keynes MK7 6AA

England
Author's current address:

Department of Mathematics

King's College

Strand

London WC2R 2LS

England 